\title{
Optimal implantation site of orthodontic micro- screws in the mandibular anterior region based on CBCT
}

\section{Yannan Wang ( $\sim$ WANGYANNAN0327@163.com )}

Chinese PLA General Hospital https://orcid.org/0000-0002-5063-6838

\section{Shi Quan}

Chinese PLA General Hospital

Juan Xu

Chinese PLA General Hospital

\section{Feng Wang}

Sixth Medical Center of PLA General Hospital

\section{Research article}

Keywords: $\mathrm{CBCT}$, mandibular anterior region, micro-screws

Posted Date: December 30th, 2019

DOI: https://doi.org/10.21203/rs.2.19717/v1

License: (c) (i) This work is licensed under a Creative Commons Attribution 4.0 International License.

Read Full License

Version of Record: A version of this preprint was published at Frontiers in Physiology on May 20th, 2021. See the published version at https://doi.org/10.3389/fphys.2021.630859. 


\section{Abstract}

Background To determine the optimal implantation site of orthodontic micro-screws based on cone beam computed tomography (CBCT) analysis in the mandibular anterior tooth region, provide a theoretical basis for orthodontic implant placement and improve post-implantation stability.

Methods Forty patients who underwent CBCT scanning were selected for this study. CBCT scanning was applied to measure the interradicular distance, buccolingual dimension, labial cortical bone thickness and lingual cortical bone thickness between mandibular anterior teeth at planes $2 \mathrm{~mm}, 4 \mathrm{~mm}, 6 \mathrm{~mm}$ and 8 $\mathrm{mm}$ below the alveolar ridge crest. The data were measured and collected to obtain a comprehensive evaluation of the specific site conditions of the alveolar bone.

Results The interradicular distance, buccolingual dimension and labial cortical bone thickness between the mandibular anterior teeth were positively correlated with the distance below the alveolar ridge crest (below $8 \mathrm{~mm}$ ). The interradicular distance, buccolingual dimension, labial cortical bone thickness and lingual cortical bone thickness were all greater than those in other areas between the lateral incisor root and canine incisor root $4 \mathrm{~mm}, 6 \mathrm{~mm}$, and $8 \mathrm{~mm}$ below the alveolar ridge crest.

Conclusion The area between the lateral incisor root and the canine incisor root in planes $4 \mathrm{~mm}, 6 \mathrm{~mm}$, and $8 \mathrm{~mm}$ from the alveolar ridge crest can be used as safe sites for implantation, while $8 \mathrm{~mm}$ below the alveolar ridge crest can be the optimal implantation site. An optimal implantation site can be $8 \mathrm{~mm}$ below the alveolar ridge crest between the lateral incisor root and the canine incisor root.

\section{Background}

Deep overbites of the anterior teeth are a common type of malocclusion in the clinic. Most patients with supra-erupted mandibular incisors, especially those with overdeveloped mandibular alveolar bone, usually need to have the anterior teeth intruded to improve the malocclusion ${ }^{[1]}$. Clinical devices used to intrude the anterior teeth include flat bite planes, utility arches and reverse curve arch wires, but these methods may result in obvious foreign body sensations, long chairside operation time, mandibular molar elongation and proclination, etc. In recent years, increasing numbers of orthodontists have chosen microscrews, which could be absolutely stable anchors for moving teeth to ideal locations, as stationary anchors, especially for adult patients who prefer not to wear extraoral appliances ${ }^{[2]}$. Micro-screws have many advantages, such as flexible implant sites, ease of removal, less malaise after operation, and immediate loading by mechanica fit between the bone surface and screw body ${ }^{[3]}$. Micro-screws also have a good curative effect for bimaxillary protrusions, gummy smiles and deep overbites.

The effectiveness of orthodontics is dependent on the sustained and effective stability of the anchors, and factors that influence implant anchoring can be mainly classified into categories such as patient characteristics (age, gender, smoking habits, dental hygiene condition), surgical operation (mechanical 
injury to bones from implant surgery, thermal injury), and micro-screw characteristics (diameter, length, implant angle, implant site bone density, loading force duration) ${ }^{[4-6]}$.

Operations to implant anchors are simple, but there is still a certain risk of surgical complications. In particular, due to the narrow interfurca distance, when the implant site is near the dental root or has a particular direction of deviation, the dental capsule and dental root may be injured, causing inflammation and even micro-screw loosening; therefore, it is very important to select the implant site accurately ${ }^{[7]}$. To effectively use implant anchors, many researchers have made preoperative assessments of micro-screw implant sites using CBCT. Their results have shown that based on $\mathrm{CBCT}$, cortical bone thickness and sclerotin conditions could be effectively assessed, and the percentage of successfully implanted microscrews could be effectively increased ${ }^{[8,9]}$.

This research analyzes the safety margin and relative stable area of micro-screw implant sites for mandibular anterior teeth, based on the anatomic form measured by CBCT, to improve the initial stability of implant anchors and effectiveness of orthodontic treatments and to provide a reference for clinical operations.

\section{Methods}

1 Subjects Forty patients who underwent CBCT scanning in our department from January 2017 to June 2018 were selected for this study, with a male to female ratio of 1:1 and an age range of 20 to 40 years. All subjects voluntarily signed the informed consent, and the study protocol was reviewed and approved by the ethics committee of the PLA General Hospital.

The inclusion criteria were as follows: 1 ) no orthodontic treatment history; 2) bilateral symmetry of the jaw bones; 3) good dental hygiene and no untreated or controlled periodontal disease or diseases of the oral mucosa; 4) overall health with no systemic disease that may influence bone metabolism; 5) X-ray film showing mandibular anterior teeth without obvious root resorption and with normal root morphology; (6) presence of mandibular tooth 3-3.

The exclusion criteria were as follows: 1 ) anterior tooth crowding greater than $1.0 \mathrm{~mm}$ or severe rotation and declination; 2) root resorption or deformity; 3 ) periodontal disease in the mandibular anterior region or alveolar bone defect resulting from other factors; 4) bone metabolism disease.

2 CBCT Scans of all patients were performed while the patients were held in a standing position. The head was adjusted to make the facial midline consistent with the median sagittal plane indication line and the orbitomeatal plane consistent with the horizontal plane indication lines. The lips were closed naturally, and the patient was asked to perform eupnea with no deglutition. All CBCT scans was performed with the same parameters, and three-dimensional reconstruction was performed using NNT Viewer v5.3 software with the scanning data, allowing observation of the coronal plane, sagittal plane, and horizontal plane in the mandibular anterior region. The steps were as follows: first, angles were adjusted to align the horizontal axis and the crest of the ridge between two dental roots; then, the sagittal 
plane was adjusted to align with the long axis of the teeth to measure the buccolingual alveolar bone thickness and cortical bone thickness; finally, the coronal plane was adjusted to align with the crest of the ridge to measure the interradicular distances between dental roots. The scanned data were saved under the DICOM 3.0 standard and were analyzed and three-dimensionally reconstructed by a CBCT image analyzer, as shown in Fig. 1.

Fig. 1 CBCT scanning and 3D reconstruction: A horizontal view; B coronal view; C sagittal view; D threedimensional reconstruction

3 Measurement method CBCT scanning was applied to measure the interradicular distances between neighboring roots, buccolingual dimensions, labial cortical bone thickness and lingual cortical bone thickness between maxillary anterior teeth at planes $2 \mathrm{~mm}, 4 \mathrm{~mm}, 6 \mathrm{~mm}$ and $8 \mathrm{~mm}$ below the alveolar ridge crest of all patients, as shown in Fig. 2 and Fig. 3. The measurement was repeated after two weeks, and the average was taken between the two measurements.

Fig. 2 CBCT measurement: interradicular distance in coronal view;

Fig. 3 CBCT measurement: buccolingual dimension, labial cortical bone thickness and lingual cortical bone thickness in sagittal view

4 Statistical analysis SPSS 19.0 software was used for the statistical analysis. The measurement data (represented as the mean $\pm S D$ ) were analyzed with repeated-measures ANOVA using position and distance as independent variables; pairwise comparison LSD t-tests were used for post hoc analysis for position, and pairwise difference t-test was used for post hoc analysis for distance. $\mathrm{P} \otimes 0.05$ indicates statistically significant differences.

\section{Results}

\section{Interradicular distances between dental roots}

The scanning data of alveolar bone interradicular distances in the mandibular anterior tooth region are listed in table 1. The overall comparisons (repeated-measures ANOVA) show that the effects of position, distance, and their interaction are all significant $(\mathrm{P}<0.05)$. Detailed pairwise comparison results coupled with the data show that the interradicular distance between the mandibular anterior teeth is positively correlated with the distance below the alveolar ridge crest. Between the lateral incisor and canine, at $8 \mathrm{~mm}$ below the alveolar ridge crest, interradicular distances reached a maximum value of $2.77 \mathrm{~mm}$. Between the central incisor and lateral incisor, at $2 \mathrm{~mm}$ below the alveolar ridge crest, interradicular distances reached a minimum value of $1.38 \mathrm{~mm}$. 
Table 1 CBCT results of the mesiodistal dimension in the mandibular anterior tooth region $(\mathrm{mm}, \pm \mathrm{s})$

\begin{tabular}{cccc}
\hline position & $1-1$ & $1-2$ & $2-3$ \\
\hline $2 \mathrm{~mm}$ & $1.47 \pm 0.51$ & $1.38 \pm 0.30$ & $1.95 \pm 0.51 \mathrm{ab}$ \\
$4 \mathrm{~mm}$ & $1.55 \pm 0.63$ & $1.49 \pm 0.71$ & $2.17 \pm 0.65 \mathrm{abt}$ \\
$6 \mathrm{~mm}$ & $1.68 \pm 0.70 \mathrm{t}$ & $1.47 \pm 0.49 \mathrm{t}$ & $2.45 \pm 0.70 \mathrm{abt}$ \\
$8 \mathrm{~mm}$ & $1.98 \pm 0.90 \mathrm{t}$ & $1.60 \pm 0.57 \mathrm{at}$ & $2.77 \pm 0.86 \mathrm{abt}$ \\
\hline Overall & \multicolumn{3}{c}{ (HF coefficient: 0.678$)$} \\
Position F, P & \multicolumn{3}{c}{$37.194,0.000$} \\
Distance F, P & \multicolumn{3}{c}{$41.925,0.000$} \\
Interaction F, P & \multicolumn{3}{c}{$6.773,0.004$} \\
\hline
\end{tabular}

a $\mathrm{P} \square 0.05$, vs $1-1$; b $\mathrm{P} \square 0.05$, vs $1-2$; t $\mathrm{P} \square 0.05$, vs $2 \mathrm{~mm}$

HF: Huynh-Feldt

central incisors: 1-1, central incisor and lateral incisor: 1-2, lateral incisor and canine teeth: $2-3$

\section{Buccolingual dimension between dental roots}

The results listed in table 2 for the analysis of the buccolingual dimension show that the effects of position, distance, and their interaction are all significant $(\mathrm{P}<0.05)$. A detailed pairwise comparison coupled with the data shows that the buccolingual dimension between the mandibular anterior teeth is positively correlated with the distance below the alveolar ridge crest. Between the lateral incisor and canine tooth, at $4 \mathrm{~mm}$ below the alveolar ridge crest, the buccolingual dimension reached a maximum value of $7.04 \mathrm{~mm}$. Between the two central incisor teeth, at $2 \mathrm{~mm}$ below the alveolar ridge crest, the buccolingual dimension reached a minimum value of $4.99 \mathrm{~mm}$.

Tab. 2 CBCT results of the buccolingual dimension in the mandibular anterior tooth region $(\mathrm{mm}, \pm \mathrm{s})$ 


\begin{tabular}{cccc}
\hline position & $1-1$ & $1-2$ & $2-3$ \\
\hline $2 \mathrm{~mm}$ & $4.99 \pm 0.75$ & $5.88 \pm 0.81 \mathrm{a}$ & $6.64 \pm 0.80 \mathrm{ab}$ \\
$4 \mathrm{~mm}$ & $5.29 \pm 0.94 \mathrm{t}$ & $6.17 \pm 0.94 \mathrm{at}$ & $7.04 \pm 0.92 \mathrm{abt}$ \\
$6 \mathrm{~mm}$ & $5.40 \pm 0.80 \mathrm{t}$ & $5.91 \pm 0.82 \mathrm{a}$ & $6.76 \pm 0.98 \mathrm{ab}$ \\
$8 \mathrm{~mm}$ & $5.76 \pm 0.90 \mathrm{t}$ & $5.95 \pm 0.87$ & $6.82 \pm 1.12 \mathrm{ab}$ \\
\hline Overall & \multicolumn{3}{c}{ (HF coefficient: 0.885) } \\
Position F, P & \multicolumn{3}{c}{$125.994,0.000$} \\
Distance F, P & \multicolumn{3}{c}{$4.638,0.019$} \\
Interaction F, P & \multicolumn{3}{c}{$16.614,0.000$} \\
\hline
\end{tabular}

a $\mathrm{P} \square 0.05$, vs $1-1$; b $\mathrm{P} \square 0.05$, vs 1-2; t $\mathrm{P} \square 0.05$, vs $2 \mathrm{~mm}$

\section{Labial cortical bone thickness between dental roots}

The results listed in table 3 for the analysis of labial cortical bone thickness show that the effects of both position and distance are significant $(\mathrm{P}<0.05)$, while their interaction is not significant. The labial cortical bone thickness between the mandibular anterior teeth is positively correlated with the distance below the alveolar ridge crest. Between the lateral incisor and canine, at $8 \mathrm{~mm}$ below the alveolar ridge crest, the labial cortical bone thickness reached a maximum value of $1.52 \mathrm{~mm}$. Between the two central incisor teeth, at 2 $\mathrm{mm}$ below the alveolar ridge crest, the labial cortical bone thickness reached a minimum value of $0.97 \mathrm{~mm}$.

Tab. 3 CBCT results of labial cortical bone thickness in the mandibular anterior tooth region $(\mathrm{mm}, \pm \mathrm{s})$

\begin{tabular}{cccc}
\hline From the alveolar bone crest & $1-1$ & $1-2$ & $1-3$ \\
\hline $2 \mathrm{~mm}$ & $0.97 \pm 0.25$ & $0.99 \pm 0.26$ & $1.23 \pm 0.27 \mathrm{ab}$ \\
$4 \mathrm{~mm}$ & $0.97 \pm 0.26$ & $0.97 \pm 0.22$ & $1.25 \pm 0.30 \mathrm{ab}$ \\
$6 \mathrm{~mm}$ & $0.98 \pm 0.29$ & $1.05 \pm 0.23$ & $1.33 \pm 0.27 \mathrm{abt}$ \\
$8 \mathrm{~mm}$ & $1.18 \pm 0.35 \mathrm{t}$ & $1.21 \pm 0.30 \mathrm{t}$ & $1.52 \pm 0.30 \mathrm{abt}$ \\
\hline Overall & \multicolumn{3}{c}{ (HF coefficient: 0.167$)$} \\
Position F, P & \multicolumn{3}{c}{$31.160,0.000$} \\
Distance F, P & \multicolumn{3}{c}{$27.097,0.000$} \\
Interaction F, P & \multicolumn{3}{c}{$0.577,0.733$} \\
\hline
\end{tabular}

a $\mathrm{P} \square 0.05$, vs $1-1 ; \mathrm{b} \mathrm{P} \square 0.05$, vs $1-2$; t $\mathrm{P} \square 0.05$, vs $2 \mathrm{~mm}$

\section{Lingual cortical bone thickness between dental roots}


Only the effect of position was significant $(\mathrm{P}<0.05)$ in the analysis of lingual cortical bone thickness, as shown in table 4 . The lingual cortical bone thickness between the mandibular anterior teeth is positively correlated with the distance below the alveolar ridge crest. The lingual cortical bone thickness between the lateral incisor and canine is larger than the bone thicknesses between all other teeth at the same plane of distance below the alveolar ridge crest. At $8 \mathrm{~mm}$ below the alveolar ridge crest, the thickness reached a maximum value of $2.30 \mathrm{~mm}$. Between the central incisor and lateral incisor, at $2 \mathrm{~mm}$ below the alveolar ridge crest, the lingual cortical bone thickness reached a minimum value of 1.27 $\mathrm{mm}$.

Tab. 4 CBCT results of lingual cortical bone thickness in the mandibular anterior tooth region $(\mathrm{mm}, \pm \mathrm{s})$

\begin{tabular}{cccc}
\hline From the alveolar bone crest & $1-1$ & $1-2$ & $1-3$ \\
\hline $2 \mathrm{~mm}$ & $1.40 \pm 0.29$ & $1.27 \pm 0.23$ & $1.74 \pm 0.41 \mathrm{ab}$ \\
$4 \mathrm{~mm}$ & $1.51 \pm 0.32 \mathrm{t}$ & $1.36 \pm 0.27$ & $1.98 \pm 0.45 \mathrm{abt}$ \\
$6 \mathrm{~mm}$ & $1.64 \pm 0.28 \mathrm{t}$ & $1.50 \pm 0.24 \mathrm{t}$ & $2.14 \pm 0.43 \mathrm{abt}$ \\
$8 \mathrm{~mm}$ & $1.78 \pm 0.34 \mathrm{t}$ & $1.78 \pm 0.31 \mathrm{t}$ & $2.30 \pm 0.43 \mathrm{abt}$ \\
\hline Overall & (HF coefficient: 0.938$)$ \\
Position F, P & \multicolumn{2}{c}{$74.144,0.000$} \\
Distance F, P & \multicolumn{3}{c}{$75.815,0.179$} \\
Interaction F, P & \multicolumn{3}{c}{$2.384,0.033$} \\
\hline
\end{tabular}

a $\mathrm{P} \square 0.05$, vs 1-1; b $\mathrm{P} \square 0.05$, vs 1-2; t $\mathrm{P} \square 0.05$, vs $2 \mathrm{~mm}$

\section{Discussion}

The implantation of anchors into the anterior mandibular teeth is highly efficient, has a good corrective effect, is unlikely to damage important anatomical structures, and can well control the torque ${ }^{[10]}$. Common oral deformities, such as deep overbites and bimaxillary protrusion, directly influence patients' facial morphologies. Deep overbites resulting from mandibular anterior alveolar bone overdevelopment are normally corrected by lowering the anterior teeth. For patients with bimaxillary protrusion, adduction and reduction of the anterior teeth can effectively improve the facial shape. Strong anchoring is fundamental to ensure anterior teeth adduction. The use of effective anchors has become an important method for correcting bimaxillary protrusions after the extraction gap is closed ${ }^{[11,12]}$.

Orthodontic micro-screws have become popular research tools for scholars at home and abroad because of their small size, high comfort, simple implantation method, shortened treatment time and ability to meet the requirements of absolute anchoring. It is difficult to rely on imaging methods to evaluate the 
safety of sites for micro-screw implanting. Conventional X-ray film measurement can result in severe distortions and overlapping dental images, often resulting in failure to obtain cross-sections between roots, and cannot be used to measure bone thickness. In contrast, CBCT yields high-resolution images with a clear field of view and has high scanning efficiency, requires a low radiation dose, and results in few scanning artifacts. CBCT can be used for effective evaluation of the alveolar bone condition at the site of the implanted micro-screw and the positional relationship with adjacent anatomical structures, making it possible to avoid damage to important anatomical structures and provide a reference for clinical applications ${ }^{[13]}$. In addition, CBCT scans can yield spatial information about the maxillofacial region, allowing three-dimensional images to be reconstructed by a computer to perform measurements in the maxillofacial region ${ }^{[14,15]}$. Studies have shown that the stability of the micro-screw can be influenced by the choice of the implantation site, the bone condition at the implant site and the distance from the adjacent teeth. The application of $\mathrm{CBCT}$ will increase the accuracy of implantations and reduce the failure rate $e^{[16,17]}$.

Micro-screw implants are generally suitable for adult patients. Because the bone tissue of juvenile patients is in the active phase, the reconstruction of the bone tissue after loading is unstable, and bone resorption is enhanced. The bone tissue of adults is relatively stable, and bone reconstruction is gentle after implantation of micro-screws, thus improving the stability of the planting anchors. Conventional implantation sites for micro-screws are generally selected between two roots ${ }^{[18]}$. For the anterior region, micro-screws are generally used to solve problems such as mandibular protrusion and deep lamination. Therefore, the conventional implantation site is between the two central incisors, between the central incisors and the lateral incisors, or between the lateral incisors and the canines ${ }^{[19]}$. According to the data analysis of this experiment, the interradicular distance, buccolingual dimension and buccal lingual bone thickness between maxillary anterior teeth were positively correlated with distances below the alveolar ridge crest below $8 \mathrm{~mm}$. Generally, between 2-3, the interradicular distance is the widest (approximately $2.77 \mathrm{~mm}$ ) at $8 \mathrm{~mm}$ below the alveolar ridge crest. However, the choice of micro-implant diameter is often limited in the clinic. If the diameter is too large, cracks will occur in the cortical bone, which will affect stability. Therefore, when micro-screws are implanted in the mandible, it is recommended for implants generally selected in the clinic to have a diameter between $1.0 \mathrm{~mm}$ and $2 \mathrm{~mm}$. Between 2-3, at both $4 \mathrm{~mm}$ and $8 \mathrm{~mm}$ below the alveolar ridge crest, the buccolingual dimensions are wide, with dimensions of approximately $7.04 \mathrm{~mm}$ and $6.82 \mathrm{~mm}$, respectively. Therefore, the length of micro-screws should not exceed $6 \mathrm{~mm}$ in clinical practice ${ }^{[20]}$; otherwise, due to its thickness and interradicular distance, the alveolar bone may be damaged by the micro-screw. Furthermore, if the micro-screws are too close to the root, they will cause loosening and shedding, resulting in failure of the implantation surgery and damage to other important anatomical structures due to incorrect positioning. Therefore, the micro-screw should be positioned in the central area between the two roots to ensure the continuity and stability of the anchor $^{[21-23]}$. In clinical practice, it is necessary to consider the proper safety range of both the alveolar bone and the soft tissue. The closer the implant anchor is to the edge of the lip mucosa, the more friction is encountered, and the more difficult it is to maintain oral hygiene, causing the planting anchor to fall off due to inflammation ${ }^{[24,25]}$. At $2 \mathrm{~mm}$ below the alveolar ridge crest, the interradicular distance and 
buccolingual dimension between the two central incisors are $2.700 \mathrm{~mm}$ and $4.99 \mathrm{~mm}$, respectively, and those between the central incisors and lateral incisors are $1.38 \mathrm{~mm}$ and $5.88 \mathrm{~mm}$, respectively. Avoiding this area during clinical operation is recommended.

The mechanism of implant anchorage correction for a deep overbite is mainly achieved by the depression of the lower anterior teeth. Studies have confirmed that desired results can be achieved in patients with deep overbite implants with anchors implanted between the two roots of the mandible, using a $100 \mathrm{~g}$ force for 3 to 6 months. Therefore, when implants are inserted into the mandible, the results are influenced by important factors such as the values of depression, depression time, and traction direction .

The implantation angle of micro-screws can significantly affect the stress of the cortical bone. As the implantation angle increases, the thickness of the cortical bone gradually decreases, the torque of the micro-screws increases gradually, and the shedding rate increases ${ }^{[26]}$. Some studies have shown that the distance between adjacent roots is large, requiring an implantation angle of $90^{\circ}$; when the spacing between adjacent roots is small, the initial stability of the micro-screws can be significantly improved when the implantation angle is $60^{\circ}-70^{\circ}[27]$. In addition, the thickness of the cortical bone is positively correlated with the mechanical fitting force at the implanted screw-bone joint surface. In principle, the success rate of surgeries is significantly improved when the thickness of the cortical bone at the implant site is greater than $1 \mathrm{~mm}^{[28]}$. Therefore, the patient's cortical bone should be measured before surgery. If the patient's cortical bone is thin, the appropriate implantation angle and implantation direction should be selected to improve the stability of the micro-screw. The experimental data from this study showed that between 2-3, the labial and lingual bone thicknesses were thickest $8 \mathrm{~mm}$ from the alveolar crest, and the stability was the highest; between 1-1, the buccolingual dimension was short, but the interradicular distance was still acceptable; and between 1-2, the interradicular distance was acceptable, but the buccolingual dimension was short. Caution should be taken if implants are required in this area, and it is recommended to design a suitable implantation method to improve the stability of the anchor.

Due to anatomical structural limitations, the anterior region of the mandible is rich in blood vessels for the lingual nerve tissue, and the bone in that region is weak. It is difficult to implant an anchor in this location, and an implant can easily fall off. Additionally, the buccal cortex of the mandible is thick, and the depression is more difficult to manage than is the depression of the maxillary anterior teeth. After depression, the mandibular anterior teeth are more likely to undergo root resorption. Changes in alveolar bone $8 \mathrm{~mm}$ from the alveolar crest were noted in this study, and the effects of the labial mucosa and ligaments on the experimental data were not considered. Because the implant supports the lower anterior teeth, the angle of traction may have affected the results of the study, causing the anterior labia to tilt. In addition, the low-pressure value and the depression time are also important factors affecting the results. In future studies, the effects of the condition of the patient's labial soft tissue and the direction of the traction force on anchor placement will be analyzed.

\section{Conclusions}


In summary, CBCT-based measurements of the mandibular anterior region showed that within the allowable range of the soft tissue, the interradicular distance, buccolingual dimension, and labial and lingual bone thicknesses were all wide at a plane $8 \mathrm{~mm}$ below the alveolar ridge crest. In this area, microscrews with diameters of 1-2 $\mathrm{mm}$ and lengths of no more than $6 \mathrm{~mm}$ can be selected in the clinical setting. The thickness of the labial bone is the least between 1-1, and a suitable implant method should be employed when implanting micro-screws to improve their stability. Between 1-2, at a plane $2 \mathrm{~mm}$ below the alveolar ridge crest, the interradicular distance is the shortest, approximately $1.38 \mathrm{~mm}$. If a micro-screw is to be implanted here, care should be taken to select the material with the appropriate specifications.

\section{References}

1. Ohnishi $\mathrm{H}$, Yagi $\mathrm{T}$, Yasuda $\mathrm{Y}$, et al. Mini-implant for orthodontic anchorage in a deep overbite case[J]. The Angle Orthodontist, 2005, 75(3):444-452.

2. Chen $\mathrm{CH}$, Chang C S , Hsieh C H, et al. The Use of Microimplants in Orthodontic Anchorage[J]. Journal of Oral \& Maxillofacial Surgery, 2006, 64(8):1209-1213.

3. Park H S , Jeong S H, Kwon O W. Factors affecting the clinical success of screw implants used as orthodontic anchorage[J]. Am J Orthod Dentofacial Orthop, 2006, 130(1):18-25.

4. Kuroda S , Tanaka E . Risks and complications of miniscrew anchorage in clinical orthodontics[J]. Japanese Dental Science Review, 2014, 50(4):79-85.

5. Sahoo S N, Pattanaik S, Nayak T K, et al. Evaluation of Post Operative Soft Tissue Complications of Orthodontic Mini-Implants at Different Loading Times-An in-Vivo Study[J]. Indian Journal of Public Health Research \& Development, 2018, 9(11).

6. Sfondrini M F, Gandini P, Alcozer R, et al. Failure load and stress analysis of orthodontic miniscrews with different transmucosal collar diameter[J]. Journal of the mechanical behavior of biomedical materials, 2018, 87: 132-137.

7. Mohammed $\mathrm{H}$, Wafaie $\mathrm{K}$, Rizk M Z , et al. Role of anatomical sites and correlated risk factors on the survival of orthodontic miniscrew implants: a systematic review and meta-analysis[J]. Progress in Orthodontics, 2018, 19(1).

8. Stability of miniscrews with different continuous orthodontic forces as measured by cone-beam computed tomography[J]. Orthodontic Waves, 2014, 73(2):48-54.

9. Qiu L L , Li S , Bai Y X . [Preliminary safety and stability assessment of orthodontic miniscrew implantation guided by surgical template based on cone-beam CT images].[J].

Zhonghuakouqiangyixuezazhi = Zhonghuakouqiangyixuezazhi = Chinese journal of stomatology, 2016, 51(6):336.

10. Ishihara $Y$, Kuroda $S$, Sugawara $Y$, et al. Indirect usage of miniscrew anchorage to intrude overerupted mandibular incisors in a Class II patient with a deep overbite[J]. American Journal of Orthodontics and Dentofacial Orthopedics, 2013, 143(4):S113-S124. 
11. Mimura $\mathrm{H}$. Treatment of severe bimaxillary protrusion with miniscrew anchorage: treatment and complications[J]. Australian orthodontic journal, 2008, 24(2):156-163.

12. Chen $G$, Teng F , Xu T M . Distalization of the maxillary and mandibular dentitions with miniscrew anchorage in a patient with moderate Class I bimaxillary dentoalveolar protrusion[J]. Am J Orthod Dentofacial Orthop, 2016, 149(3):401-410.

13. Poon $Y C$, Chang H P , Tseng Y C , et al. Palatal bone thickness and associated factors in adult miniscrew placements: A cone-beam computed tomography study[J]. The Kaohsiung Journal of Medical Sciences, 2015, 31(5):265-270.

14. Min K I, Kim S C, Kang K H, et al. Root proximity and cortical bone thickness effects on the success rate of orthodontic micro-implants using cone beam computed tomography[J]. The Angle Orthodontist, 2012, 82(6):1014-1021.

15. Dinizfreitas M , Seoaneromero J , M. FernándezVarela, et al. Cone Beam Computed Tomography evaluation of palatal bone thickness for miniscrew placement in Down's syndrome[J]. Archives of Oral Biology, 2015, 60(9):1333-1339.

16. Yaqi $D$, Yannan $S$, Tianmin $X$. Evaluation of root resorption after comprehensive orthodontic treatment using cone beam computed tomography (CBCT): a meta-analysis[J]. BMC Oral Health, 2018, 18(1):116-.

17. Tepedino M, Cornelis M A, Chimenti C, et al. Correlation between tooth size-arch length discrepancy and interradicular distances measured on $\mathrm{CBCT}$ and panoramic radiograph: an evaluation for miniscrew insertion[J]. Dental press journal of orthodontics, 2018, 23(5): 39. e1-39. e13.

18. Chang H P , Tseng Y C . Miniscrew implant applications in contemporary orthodontics[J]. The Kaohsiung Journal of Medical Sciences, 2014, 30(3):111-115.

19. Ludwig B , Glasl B , Kinzinger G S , et al. Anatomical guidelines for miniscrew insertion: Vestibular interradicular sites[J]. Journal of Clinical Orthodontics Jco, 2011, 45(3):165-73.

20. SarulM , Minch L , Park H S , et al. Effect of the length of orthodontic mini-screw implants on their long-term stability: $\backslash$, A prospective study[J]. The Angle Orthodontist, 2015, 85(1):33-38.

21. Shigeeda, Toru. Root proximity and stability of orthodontic anchor screws[J]. Journal of Oral Science, 2014, 56(1):59-65.

22. Cornelis M A, Scheffler N R, Clerck H J D , et al. Systematic review of the experimental use of temporary skeletal anchorage devices in orthodontics[J]. American Journal of Orthodontics \& Dentofacial Orthopedics, 2007, 131(4-supp-S):0-0.

23. Xiang C , Xiang-Feng Z , Qian-Qian H , et al. Evaluation of the changes of alveolar bone around the upper incisors after retraction with mini implant anchorage using cone-beam CT[J]. Shanghai Journal of Stomatology, 2018.

24. Michele T , Cattaneo P M , Francesco M , et al. Average interradicular sites for miniscrew insertion: should dental crowding be considered?[J]. Dental Press Journal of Orthodontics, 2017, 22(5):90-97.

25. Aras I , Tuncer A V . Comparison of anterior and posterior mini-implant-assisted maxillary incisor intrusion: $\backslash$ r, Root resorption and treatment efficiency[J]. The Angle Orthodontist, 2016:085015-571.1. 
26. Rodriguez J C, Suarez F, Chan H L , et al. Implants for orthodontic anchorage: success rates and reasons of failures.[J]. Implant Dentistry, 2014, 23(2):155.

27. Yan Y Q , Gao Y M . [A clinical study on the stability of miniscrew anchorage in orthodontics][J]. Shanghai Journal of Stomatology, 2011, 20(5):540.

28. Zheng $\mathrm{J}$, Yeke $\mathrm{W}$, Wenlu $\mathrm{J}$, et al. Factors Affecting the Clinical Success Rate of Miniscrew Implants for Orthodontic Treatment[J]. The International Journal of Oral \& Maxillofacial Implants, 2016, 31(4):835-841.

\section{Declarations}

\section{Ethics approval and consent to participate}

The study protocol was reviewed and approved by the ethics committee of the PLA General Hospital.

\section{Consent for publication}

All subjects voluntarily signed the informed consent.

\section{Availability of data and materials}

The datasets used and/or analysed during the current study are available from the corresponding author on reasonable request.

\section{Competing interests}

The authors declare that they have no competing interests.

\section{Funding}

Not applicable.

\section{Authors' contributions}

YW and SQ designed the study in consultation with JX and FW; all authors assisted with sample collection; YW and SQ conducted data acquisition and analysis; YW drafted the article and revised with 
other authors. All authors have read and approved the manuscript.

\section{Acknowledgements}

Not applicable

\section{Figures}

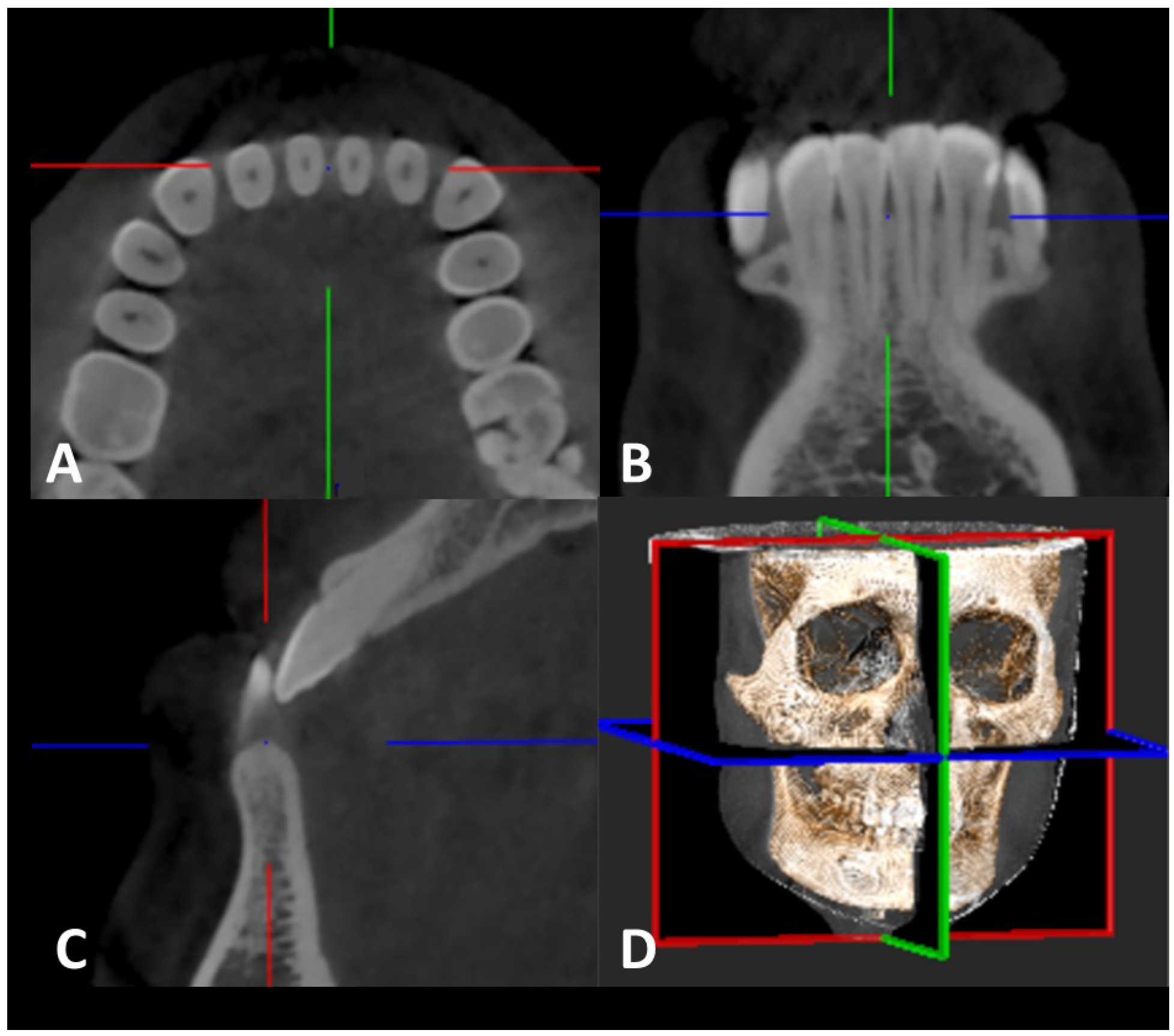

Figure 1 
CBCT scanning and 3D reconstruction: A horizontal view; B coronal view; C sagittal view; $D$ threedimensional reconstruction

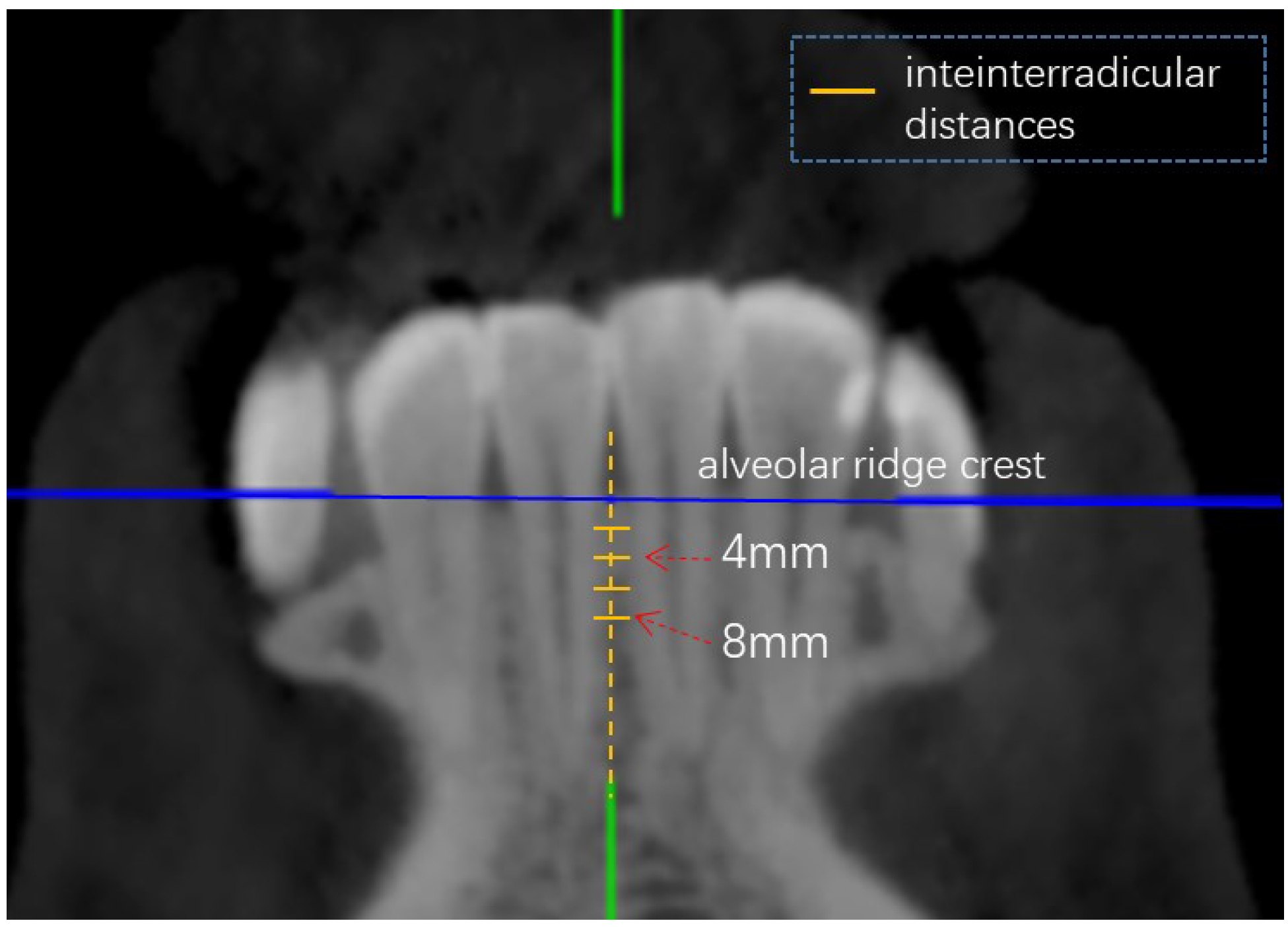

Figure 2

CBCT measurement: interradicular distance in coronal view 


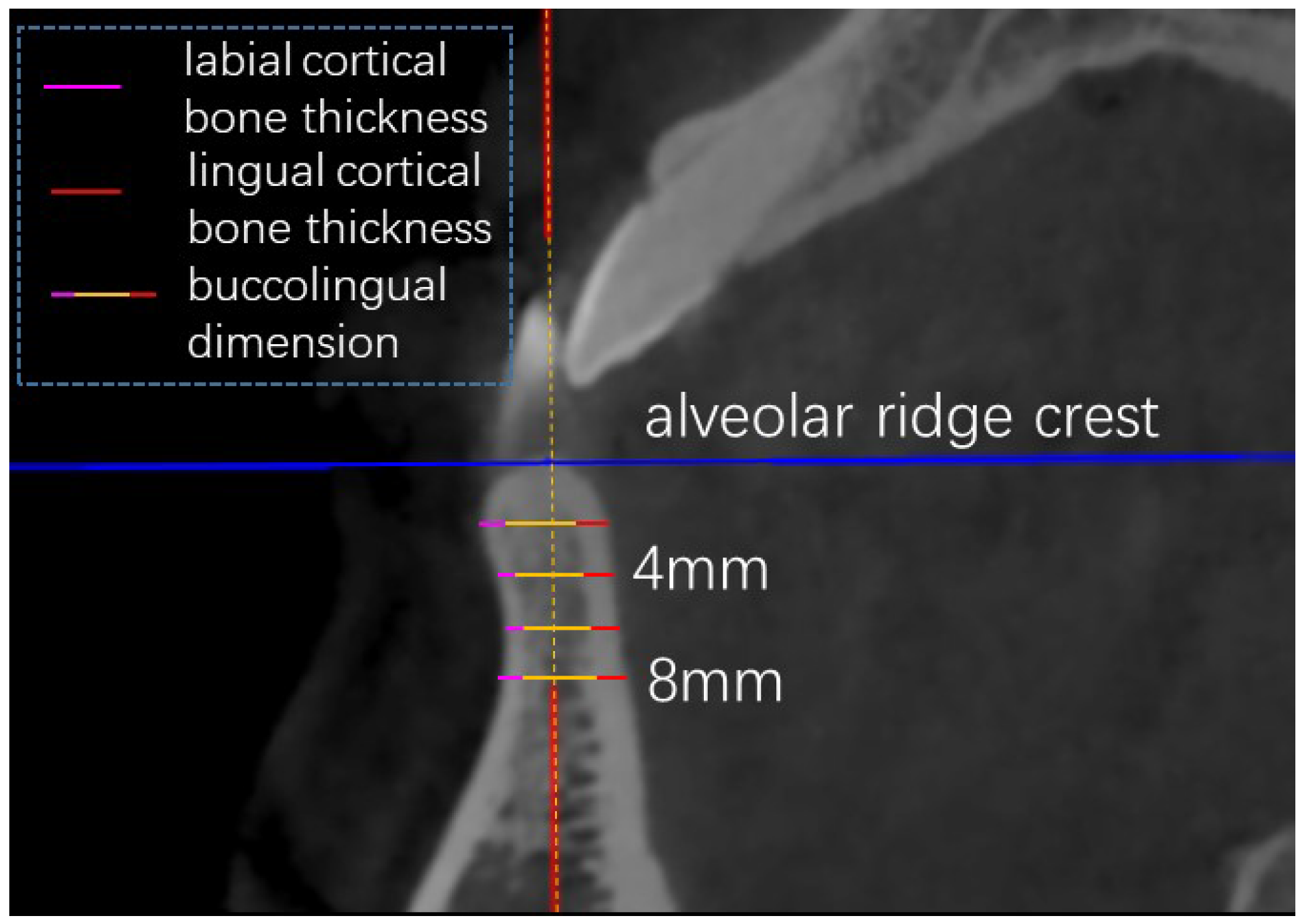

Figure 3

CBCT measurement: buccolingual dimension, labial cortical bone thickness and lingual cortical bone thickness in sagittal view 